Article

\title{
Optimization of Baicalin, Wogonoside, and Chlorogenic Acid Water Extraction Process from the Roots of Scutellariae Radix and Lonicerae japonicae Flos Using Response Surface Methodology (RSM)
}

\author{
Jichang Li ${ }^{1,2}$, Rui Wang ${ }^{1}$, Zunlai Sheng ${ }^{1}$, Zhiyong $\mathrm{Wu}^{1}{ }^{1}$, Chunli Chen ${ }^{1, *}$ and \\ Muhammad Ishfaq ${ }^{1, * \mathbb{D}}$ \\ 1 College of Veterinary Medicine, Northeast Agricultural University, 600 Changjiang Road, Xiangfang District, \\ Harbin 150030, China; lijichang828@sina.com (J.L.); wr6915522@126.com (R.W.); \\ shengzunlai@neau.edu.cn (Z.S.); neauwuzhiyong@163.com (Z.W.) \\ 2 Heilongjiang Key Laboratory for Animal Disease Control and Pharmaceutical Development, 600 Changjiang \\ Road, Xiangfang District, Harbin 150030, China \\ * Correspondence: chunlichen@outlook.com (C.C.); ishfaqmuhammad@neau.edu.cn (M.I.); \\ Tel.: +86-451-5519-0674 (C.C. \& M.I.); Fax: +86-451-5519-1200 (C.C. \& M.I.)
}

Received: 18 October 2019; Accepted: 11 November 2019; Published: 14 November 2019

check for updates

\begin{abstract}
In this study, a simultaneous water extraction process for baicalin, wogonoside, and chlorogenic acid has been optimized. The effect of extraction temperature, extraction time, and liquid-solid ratio was scrutinized by single factor experiments and further analyzed by Box-Behnken design (BBD) approach using response surface methodology (RSM). The extraction yield of investigated compounds was determined by high performance liquid chromatography (HPLC). Single-factor experiments and response surface analysis results revealed that the optimized conditions are: Liquid to solid ratio $25: 1(\mathrm{~mL} / \mathrm{g})$, extraction temperature $93^{\circ} \mathrm{C}$, extraction time $2.4 \mathrm{~h}$, and the extraction cycle two. Importantly, it has been noted that under the above conditions, concentrations of baicalin, wogonoside, and chlorogenic were $0.078,0.031$, and $0.013 \mathrm{mg} / \mathrm{mL}$, respectively, and the overall desirability (OD) value was 0.76 which was higher than the non-optimized conditions and the deviation from the predicted OD value was only $2.44 \%$. Conclusively, it has been suggested that the model was stable and feasible, and fit for extraction of baicalin, wogonoside, and chlorogenic acid from Scutellariae Radix and Lonicerae (L.) japonicae Flos.
\end{abstract}

Keywords: baicalin; wogonoside; chlorogenic acid; extraction process; HPLC

\section{Introduction}

Scutellariae Radix is used in various pharmacological preparations in several East Asian countries [1]. The active ingredients of this herbal plant contain several flavonoid compounds including wogonoside, baicalin, and their aglycone forms wogonin and baicalein [2]. Numerous studies have reported the anti-inflammatory, anti-oxidative, antiviral, and anti-tumor effects of baicalin [3]. Recently, baicalin bewitch the interest of scientist due to its antibacterial effect with the benefit of avoiding drug resistance, and has been used as an adjuvant drug in the treatment of hepatitis [4]. The second major active ingredient in Scutellariae Radix is wogonoside, reported for its excellent anti-allergic, antioxidant, anti-inflammatory, and hepatoprotective activities [5]. The anti-inflammatory activity of wogonoside has been found due to the inhibition of TNF- $\alpha$ and IL-6 production in LPS-stimulated RAW264.7 cells [6]. Hence, it is important to develop and optimize a comprehensive extraction process for the two flavones (baicalin and wogonoside). Previous studies reported that Lonicerae (L.) japonicae Flos has 
broad spectrum of pharmacological activities including antipyretic, anti-inflammatory, antiviral, and antibacterial activities. L. japonicae Flos has also been used for the treatment of pneumonia, swelling, and even used in herbal tea, toothpaste, and cosmetics [7,8]. Importantly, chlorogenic acid (polyphenol) is the biological active component of L. japonicae Flos [9]. However, knowledge regarding the extraction process of chlorogenic acid is limited. Therefore, a reliable and efficient method is needed to extract chlorogenic acid from L. japonicae Flos.

Extraction techniques are mainly divided into modern and traditional methods [10]. Traditional extraction methods including reflux, maceration, percolation, and soxhlet extraction [11]. While, supercritical fluid extraction (SFE), microwave assisted extraction (MAE), pressurized liquid extraction (PLE), and ultrasound assisted extraction (UAE) methods are within the list of modern extraction methods [12,13]. Recently, researchers focused on modern methods to be faster, more efficient, and consume less organic solvent [14]. On the contrary, in the current study, we focused on traditional water extraction process for the simultaneous extraction of baicalin, wogonoside, and chlorogenic acid. The current method is inexpensive which consumes less energy and machinery as compared to modern extraction methods. The extract of Scutellariae Radix and L. japonicae Flos have synergistic pharmacological activities, and therefore used together in traditional Chinese medicine. The objective of the current study is to optimize a simultaneous water extraction process for baicalin, wogonoside, and chlorogenic acid from Scutellariae Radix and L. japonicae Flos. The effect of extraction temperature, extraction time, and liquid-solid ratio was tested by single-factor experiments, and the optimized conditions were further validated through Box-Behnken design (BBD) and response surface methodology (RSM) approach. The data showed that the experimental values were accurate and the model applied for the extraction process was fit and reliable. Nevertheless, the isolated products (baicalin, wogonoside, and chlorogenic acid) were confirmed and quantified by HPLC.

\section{Materials and Methods}

\subsection{Ethical Statement}

All the experimental protocols are performed in accordance with the guidelines and approved protocols of Northeast Agricultural University.

\subsection{Samples and Chemicals Reagents}

Wogonoside standard (batch no. 112002-201702, purity 98.5\%) and baicalin (batch no. 110715-201720, purity 93.5\%) were purchased from Chinese veterinary medicine supervision center (Beijing, China). National Institutes for Food and Drug Control (Beijing, China) provided chlorogenic acid (batch no. Z0261702, purity 98.3\%). Milli-Q academic water purification system (Millipore, Bedford, MA, USA) provided purified distilled water. L. japonicae Flos (batch no. 16122301) and Scutellariae Radix (batch no. 17081201) were purchased from Runhe Chinese medicine processing plant Ltd. (Heilongjiang, China). These plants were authenticated and identified by Heilongjiang University of Chinese Medicine (Heilongjiang, China). The specimens (Accession no; 1009012ch and $1009013 \mathrm{ch}$ ) were placed in the herbarium of Northeast Agricultural University (College of Veterinary Medicine). Ethanol, methanol, and phosphoric acid (HPLC grade) were purchased from Kermel Chemical Reagent Co., Ltd. (Tianjin, China).

\subsection{Preparation of Standard Solution of Baicalin, Wogonoside, and Chlorogenic Acid}

Standard substances of baicalin (20 mg), wogonoside (10 mg), or chlorogenic acid (5 mg) was accurately weighed and dissolved in methanol in a $50 \mathrm{~mL}$ volumetric flask, respectively. Standard stock solutions of baicalin $(0.4 \mathrm{mg} / \mathrm{mL})$, wogonoside $(0.2 \mathrm{mg} / \mathrm{mL})$, and chlorogenic acid $(0.1 \mathrm{mg} / \mathrm{mL})$ were prepared accordingly. 1, 2, 3, 4, and $5 \mathrm{~mL}$ of the stock solution were taken in a $10 \mathrm{~mL}$ volumetric flask, and methanol was added to obtain $0.04,0.08,0.12,0.16,0.20 \mathrm{mg} / \mathrm{mL}$ of baicalin standard solutions, 0.02 , 
$0.04,0.06,0.08,0.10 \mathrm{mg} / \mathrm{mL}$ of wogonoside standard solutions, and $0.01,0.02,0.03,0.04,0.05 \mathrm{mg} / \mathrm{mL}$ of chlorogenic acid standard solutions, respectively.

\subsection{Sample Preparation and Extraction Protocol}

The Scutellariae Radix (30 g) or L. japonicae Flos (20 g) was crushed by a grinder (AK-1000A, Wenling, China) into coarse powder of $150 \mu \mathrm{m}$. In order to get the ranges of different factors (i.e., extraction temperature, extraction time, and the liquid-solid ratio) in the single-factor test design, $3 \mathrm{~g}$ of Scutellariae Radix powder and $2 \mathrm{~g}$ of L. japonicae Flos powder were mixed and soaked together in distilled water in a conical flask, and boiled at setting temperature and time. After centrifugation (3000 rpm for $10 \mathrm{~min}$ ), supernatant was taken, and condensed to $10 \mathrm{~mL}$ in a vacuum chamber (Yiheng Technology Co., Ltd., Shanghai, China). Then, $95 \%$ ethanol $(280 \mathrm{~mL})$ was added to it and placed it in $5^{\circ} \mathrm{C}$ refrigerator for $12 \mathrm{~h}$. The mixture was then centrifuged ( $3000 \mathrm{rpm}$ for $10 \mathrm{~min}$ ) to remove the precipitate, and the supernatant was vacuum dried. Methanol was added to the dried extract up to $10 \mathrm{~mL}$. Finally, $1 \mathrm{~mL}$ was taken from the dissolved dried extract and moved to a $100 \mathrm{~mL}$ volumetric flask, and diluted with methanol to a final volume of $100 \mathrm{~mL}$, obtaining a sample solution of $5 \mathrm{mg} / \mathrm{mL}$, which was filtrated by $0.22 \mu \mathrm{m}$ membrane for high performance liquid chromatography (HPLC) analysis.

\subsection{HPLC Quantification and Method Validation}

High performance liquid chromatography (HPLC) was performed with an HPLC Acquity (Waters Alliance, USA), equipped with a 2998-UV detector (Waters, Milford, USA). An injection volume of $10 \mu \mathrm{L}$ was injected into the apparatus and a flow rate of $1 \mathrm{~mL} / \mathrm{min}$ was maintained through the column. Mobile phase is consisted of acetonitrile-water-glacial acetic acid with the ratio of 47:53:0.2 $(\mathrm{v} / \mathrm{v} / \mathrm{v})$. A detection wavelength of $275 \mathrm{~nm}$ was used for baicalin and wogonoside, while $325 \mathrm{~nm}$ was used for chlorogenic acid at a column temperature of $25^{\circ} \mathrm{C}$. Kromasil C18 $(4.6 \times 250 \mathrm{~mm}, 5 \mu \mathrm{m})$ column was employed in the process. Analysis of the data was carried out by Empower 2 software installed in a computer controlling HPLC system.

The HPLC method was validated in terms of linearity, precision, stability, reproducibility, and recovery. Working solutions $(10 \mu \mathrm{L})$ of each concentration of baicalin, wogonoside, and chlorogenic acid standards were analyzed under chromatographic conditions as stated above, and taking chromatographic peak area as the $\mathrm{Y}$ axis and the concentration of the standards as the $\mathrm{X}$ axis to draw the standard curve, the correlation coefficient ( $r$ value) was used to evaluate the linear relationship and then the range of linearity was obtained. The standard solutions in three different concentrations (low, medium, and high), i.e., baicalin $(0.04,0.12,0.20 \mathrm{mg} / \mathrm{mL})$, wogonoside $(0.02,0.06,0.10 \mathrm{mg} / \mathrm{mL})$, and chlorogenic acid $(0.01,0.03,0.05 \mathrm{mg} / \mathrm{mL})$ were determined, respectively five times in a day to validate the intra-day precision. The extract solution $(5 \mathrm{mg} / \mathrm{mL})$ was detected by HPLC (injection volume of $10 \mu \mathrm{L}$ ) at $0,2,4,8,12$, and $24 \mathrm{~h}$ at room temperature, respectively to judge the stability of the extract samples. Solutions of five batches of extract sample $(5 \mathrm{mg} / \mathrm{mL}$ ) were prepared (as stated above), and $10 \mu \mathrm{L}$ of each sample solution was injected for HPLC analysis $(n=6)$ to validate the reproducibility (inter-day precision). The peak areas of baicalin, wogonoside, and chlorogenic acid were determined, respectively and the coefficient of variation (RSD\%) values were calculated to determine stability, precision, and reproducibility. In order to examine the accuracy of the method, recovery test was carried out, five dried extract samples of known quantity were precisely weighed and baicalin, wogonoside, or chlorogenic acid standard (i.e., $1.0,0.45$, and $0.15 \mathrm{mg}$, respectively) was added and $10 \mu \mathrm{l}$ of each sample was injected for HPLC analysis and finally recovery rate was calculated.

\subsection{Single-Factor Test Design}

The effect of extraction temperature $\left(60,70,80,90\right.$, and $\left.100{ }^{\circ} \mathrm{C}\right)$, extraction time $(1,2,3,4$, and $5 \mathrm{~h})$ and the liquid-solid ratio (10:1, 15:1, 20:1, 25:1, and 30:1 ml/g) were tested. A single-factor test was performed to determine the suitable range of three factors, which was taken from the extraction 
yield ( $\mathrm{R}$ value) as the judgment index. The whole process including sample preparation and HPLC determination was repeated three times, and $\mathrm{R}$ was determined by the following equation:

$$
R=\frac{C 1}{C 2} \times 1000
$$

where $R$ represents the extraction yield ( $\mathrm{mg} / \mathrm{g}$ ) of chlorogenic acid, baicalin, or wogonoside in each gram of crude drug. $C_{1}$ represents the content of chlorogenic acid or baicalin or wogonoside in the sample solution determined by HPLC and calculated via standard curve, which is the number of milligrams of each component contained in the sample solution per milliliter, and $C_{2}$ represents the concentration of the sample solution as stated above $(5 \mathrm{mg} / \mathrm{mL})$, which is the number of milligrams of Scutellariae Radix and L. japonicae Flos in the sample solution per milliliter.

Meanwhile, in order to synthesize all the indexes into a value which can reflect the result of the total effect, the overall desirability (OD) was introduced to investigate the comprehensive effect. Each treatment was performed in triplicates. The formula is as follow:

$$
d_{i}=\frac{Y_{i}-Y_{\min }}{Y_{\max }-Y_{\min }}
$$

where $Y_{i}$ is the actual measurement value, $Y_{\min }$ and $Y_{\max }$ are the minimum and maximum of all values measured in the group. After calculating the $d_{i}$ value, the OD value is computed according to the following equation:

$$
\mathrm{OD}=\left(\mathrm{d}_{1} \times \mathrm{d}_{2} \times \cdots \times \mathrm{d}_{\mathrm{n}}\right)^{1 / \mathrm{n}}
$$

The effects of extraction temperature on the extraction yield of baicalin, wogonoside, and chlorogenic acid were investigated at liquid-solid ratio $(20: 1 \mathrm{ml} / \mathrm{g})$ and at an extraction time of $1 \mathrm{~h}$. While, the effects of extraction temperature on the extraction yield of baicalin, wogonoside, and chlorogenic acid were determined under an extraction temperature of $90^{\circ} \mathrm{C}$ and liquid-solid ratio $(20: 1 \mathrm{~mL} / \mathrm{g})$. In addition, the effects of extraction temperature on the extraction yield of baicalin, wogonoside, and chlorogenic acid were investigated under the fixed conditions of extraction temperature $\left(90^{\circ} \mathrm{C}\right)$, extraction time of $3 \mathrm{~h}$ at first extraction cycle.

\subsection{Box-Behnken Design (BBD) for Optimization of Extraction Conditions}

A 17-run Box-Behnken design (BBD) was used for the optimization of parameters which affected the extraction process of baicalin, wogonoside, and chlorogenic acid. According to single-factor test, the optimum extracting conditions were determined based on 12 factorial points and 5 axial points. OD was set as the response value, three independent variables (X1, extraction temperature; $\mathrm{X} 2$, liquid-solid ratio; $\mathrm{X} 3$, extraction time) and three levels were given the values as 1 (high), 0 (intermediate), and -1 (low) (as shown in Table 1). The equation of second-order polynomial mode is given below:

$$
Y=\beta_{0}+\sum_{i=1}^{3} \beta_{i} X_{i}+\sum_{i=1}^{3} \beta_{i i} X_{i}^{2}+\sum_{i=1}^{2} \sum_{j=i+1}^{3} \beta_{i j} X_{i} X_{j}
$$

whereas $Y$ represents the response, $\beta_{0}$ was a constant, $\beta_{i}$ (linear), $\beta_{i i}$ (quadratic) and $\beta_{i j}$ (interactive) were the coefficients; $X_{i}$ denotes independent variables. The terms $X_{i} X_{j}$ and $X_{i}^{2}$ represented the quadratic and interaction terms, respectively. 
Table 1. Factors and levels of Box-Behnken design about extraction conditions.

\begin{tabular}{ccccc}
\hline \multirow{2}{*}{ Independent Variables } & Symbols & \multicolumn{3}{c}{ Coded Levels } \\
\cline { 3 - 5 } & & $\mathbf{- 1}$ & $\mathbf{0}$ & $\mathbf{1}$ \\
\hline Extraction temperature $\left({ }^{\circ} \mathrm{C}\right)$ & $\mathrm{X}_{1}$ & 80 & 90 & 100 \\
Liquid-solid ratio $(\mathrm{mL} / \mathrm{g})$ & $\mathrm{X}_{2}$ & $20: 1$ & $25: 1$ & $30: 1$ \\
Extraction time $(\mathrm{h})$ & $\mathrm{X}_{3}$ & 2 & 3 & 4 \\
\hline
\end{tabular}

\subsection{Data Analysis}

Response surface methodology statistical analysis was carried out by Stat-Ease Design-Expert 7.0.0 (Stat-Ease Inc., Minneanopolis, MN, USA). Mean values were regarded as significantly different at ${ }^{*} p<0.05$. The experiments were performed at least in triplicates otherwise stated and the data were expressed as means $\pm \mathrm{SD}(\mathrm{n}=3)$. ANOVA (one-way analysis of variance) was used to determine statistical significance among groups followed by Tukey test through SPSS software (Version 18.0, SPSS Inc., Chicago, IL, USA).

\section{Results}

\subsection{High Performance Liquid Chromatography (HPLC) and Validation of Extraction Process}

The peaks of chlorogenic acid, baicalin, and wogonoside in the sample appeared at retention times of approximately 11, 24, and 29 min, respectively, which are matched with the corresponding standards (Figure 1A-D).
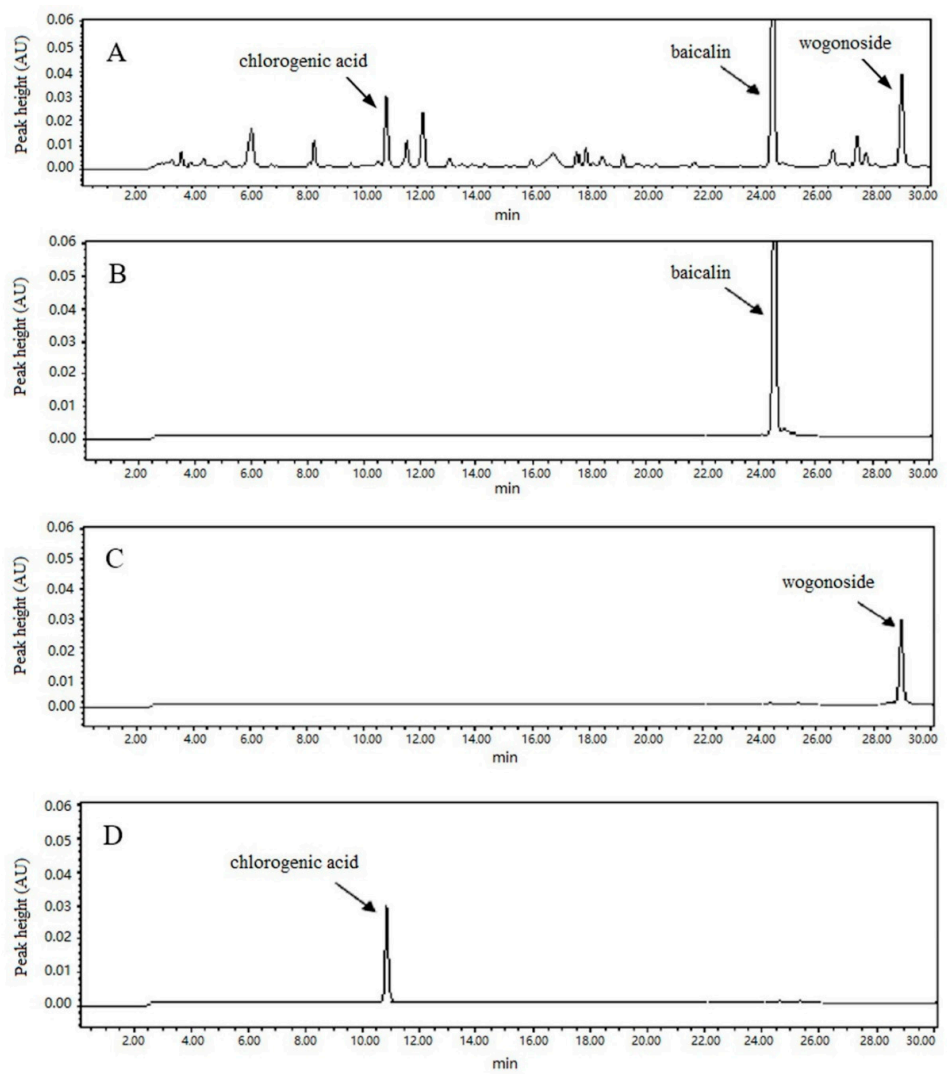

Figure 1. High performance liquid chromatography (HPLC) chromatograms of (A) mixture sample of S. baicalensis Georgi and L. japonica Thunb, and (B-D) standard solution of baicalin, wogonoside, and chlorogenic acid. 


\subsection{Standard Curve Determination and Range of Linearity}

The results obtained from regression equations of baicalin, wogonoside, and chlorogenic acid revealed a good linearity over the concentration ranged from $0.04-0.20 \mathrm{mg} / \mathrm{mL}$ (Figure 2A), 0.02-0.10 $\mathrm{mg} / \mathrm{mL}$ (Figure $2 \mathrm{~B}$ ), and $0.01-0.05 \mathrm{mg} / \mathrm{mL}$ (Figure $2 \mathrm{C}$ ), respectively.
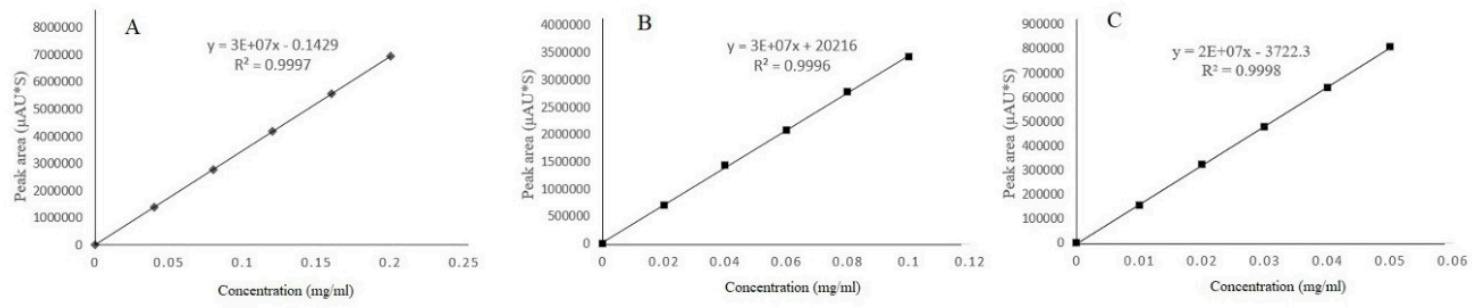

Figure 2. Standard curve of baicalin (A), wogonoside (B), and chlorogenic acid (C). Horizontal axis represents concentration of baicalin, wogonoside, and chlorogenic acid $(\mathrm{mg} / \mathrm{mL})$ and vertical axis represents peak area $\left(\left(\mu \mathrm{AU}\right.\right.$ (absorption unit) ${ }^{*} \mathrm{~S}$ (time in sec)).

\subsection{Precision Experiments}

Table 2 shows that the RSD\% values in the precision experiments are less or equal to $1.56 \%$ for three concentrations of baicalin, wogonoside, and chlorogenic acid standard solutions, respectively. The results revealed that the HPLC method was precise.

Table 2. Results of the intra-day precision $(n=5)$.

\begin{tabular}{cccc}
\hline & Concentrations $(\mathbf{m g} / \mathbf{m L})$ & Peak Areas $(\bar{x} \pm \mathbf{S D})$ & RSD $\%$ \\
\hline \multirow{2}{*}{ Baicalin } & 0.04 & $1,400,829.8 \pm 11,486.7$ & 0.82 \\
& 0.12 & $4,176,222.6 \pm 11,373.6$ & 0.27 \\
Wogonoside & 0.20 & $6,904,145.2 \pm 17,260.3$ & 0.25 \\
& 0.02 & $399,412.4 \pm 3708.5$ & 0.93 \\
Chlorogenic acid & 0.06 & $1,196,842.2 \pm 8566.3$ & 0.72 \\
& 0.10 & $2,065,237 \pm 11,709.9$ & 0.57 \\
& 0.01 & $56,175.3 \pm 876.3$ & 1.56 \\
& 0.03 & $165,526.9 \pm 2284.9$ & 1.38 \\
\end{tabular}

\subsection{Stability, Reproducibility, and Recovery of Baicalin, Wogonoside, and Chlorogenic Acid}

Table 3 indicated that the RSD $\%$ values were $1.12 \%, 1.60 \%$, and $1.14 \%$ for peak areas of baicalin, wogonoside, and chlorogenic acid, respectively, which showed that the extract samples were in good stability within $24 \mathrm{~h}$. In addition, the RSD \% values of extract samples are $2.10 \%, 2.23 \%$, and $2.91 \%$ for peak areas of baicalin, wogonoside, and chlorogenic acid, which showed that the reproducibility of the method was good (shown in Table 3). The RSD $\%$ values are $1.57 \%, 1.22 \%$, and $2.02 \%$ for baicalin, wogonoside, and chlorogenic acid, and the recovery rates are $100.69 \%, 101.68 \%$, and $99.6 \%$, respectively (Table 4).

Table 3. Stability and reproducibility of baicalin, wogonoside, and chlorogenic acid determined by HPLC.

\begin{tabular}{ccccc}
\hline & Stability & \multicolumn{3}{c}{ Reproducibility } \\
\hline & Peak Areas $(\overline{\boldsymbol{x}} \pm$ SD) & RSD & Peak Areas $(\overline{\boldsymbol{x}} \pm$ SD) & RSD \\
\hline Baicalin & $3,922,371 \pm 44,077.5$ & $1.12 \%$ & $3,969,422 \pm 83,402.7$ & $2.10 \%$ \\
Wogonoside & $1,582,977 \pm 25,327.6$ & $1.60 \%$ & $1,552,831 \pm 34,628.1$ & $2.23 \%$ \\
Chlorogenic acid & $235,760 \pm 2678.3$ & $1.14 \%$ & $236,604 \pm 6881.4$ & $2.91 \%$ \\
\hline
\end{tabular}


Table 4. Recovery rate of the samples.

\begin{tabular}{cccccc}
\hline & $\begin{array}{c}\text { Weight of Compounds in } \\
\text { the Extract Samples } \mathbf{( m g})\end{array}$ & $\begin{array}{c}\text { Weight of Standard } \\
\text { Substances Added }(\mathbf{m g})\end{array}$ & $\begin{array}{c}\text { Measured } \\
\text { Quantity (mg) }\end{array}$ & Recovery Rate $(\bar{x} \pm$ SD) \% & RSD\% \\
\hline Baicalin & $1.093 \pm 0.0262$ & 1 & $1.1937 \pm 0.019$ & $100.69 \pm 1.23$ & 1.22 \\
Wogonoside & $0.448 \pm 0.0103$ & 0.45 & $0.4938 \pm 0.003$ & $101.68 \pm 2.05$ & 2.02 \\
Chlorogenic acid & $0.153 \pm 0.0032$ & 0.15 & $0.3024 \pm 0.006$ & $99.6 \pm 1.56$ & 1.57 \\
\hline
\end{tabular}

\subsection{Single-Factor Experiments}

The effect of liquid-solid ratio, extraction time, and extraction temperature on the extraction yield of baicalin, wogonoside, chlorogenic acid and the overall evaluation of the single value OD was shown in Figure 3A-F in single-factor experiments. The extraction yield of baicalin or wogonoside was increased with the increase in temperature (Figure 3A), the OD value reflects the same trend (Figure 3B). Whereas, the extraction yield of chlorogenic acid was increased at lower temperature and decreased at higher temperature. Importantly, the extraction yield was higher from $80-90^{\circ} \mathrm{C}$ compared to other temperatures. Therefore, the center point of the extraction temperature in the response surface test was set to $90^{\circ} \mathrm{C}$ in the range of 80 to $100^{\circ} \mathrm{C}$. For the extraction time, we have observed that the extraction yield of baicalin, wogonoside, and chlorogenic acid were increased first, reached peak level at $3 \mathrm{~h}$ of extraction time and then decreased afterwards (Figure 3C). The OD value reflects that these results were significant (Figure 3D). Therefore, the center point of the extraction time in the response surface test was set to $3 \mathrm{~h}$ in the range of 2 to $4 \mathrm{~h}$. At liquid-solid ratio of 25:1 (mL/g), the OD value and extraction ratio of baicalin, wogonoside, and chlorogenic acid reached maximum value and then decreased with exceeding ratio (Figure 3E,F). However, it has been noted that the extraction yield of chlorogenic acid was less than the other two compounds. The result suggested that the appropriate liquid-solid ratio was between 20:1 and 30:1 (mL/g). Hence, a liquid-solid ratio of 25:1 (mL/g) was selected for further experiments.

\subsection{Optimization of the Extraction Parameters by Box-Behnken Design and Response Surface Methodology}

The three parameters for the extraction of baicalin, wogonoside, and chlorogenic acid were optimized by Box-Behnken design. The response values were formulated from factorial design and 17 runs, respectively (Table 5). Second-order polynomial equation (given below) was used for multiple regressions analysis for the data to calculate relativity between the response variable and test variable:

$$
\begin{gathered}
Y=-32.24569+0.64650 X_{1}+0.25780 X_{2}-0.16021 X_{3}+1.35500 E-004 X_{1} X_{2}+0.011285 X_{1} X_{3}+ \\
0.011285 X_{1} X_{3}-1.29500 E-003 X_{2} X_{3}-3.65725 E-003 X_{1}^{2}-5.29400 E-003 X_{2}^{2}-0.17628 X_{3}^{2}
\end{gathered}
$$

Model $p$-value was remarkably significant (less than 0.0001) analyzed by regression analysis of variance (Table 6), showed that the model was accurate. $X_{1}, X_{3}, X_{1} X_{3}, X_{1}^{2}, X_{2}{ }^{2}$, and $X_{3}{ }^{2}$ were significant model terms and there could be a chance of $0.01 \%$ that model ${ }^{a} F$-value increased due to noise. Lack of fit F-value (5.104) was not statistically significant $(p>0.05)$. 7.47\% chance of error was found in the lack of fit F-value due to noise signals. Therefore, the model was fit and appropriate and could be employed for experimental extraction.

Two-dimensional contour plots and three-dimensional response surface displayed in Figure 4A-F for independent variables (extraction temperature, liquid-solid ratio, and extraction time). The shape of the contour plot can reflect the strength of the interaction effect, oval shape indicates significant interaction between two interaction factors. Figure $4 \mathrm{~A}, \mathrm{~B}$ shows the effects of extraction temperature $\left(X_{1}\right)$ and extraction time $\left(X_{3}\right)$ on OD values at the liquid-solid ratio of 25:1 (mL/g). OD value first enhances and then decreases at different extraction temperature. The longer or shorter extraction time causes marked changes in OD value. The results showed that (Figure 4A) the impact of interaction was significant between extraction temperature and extraction time on OD value as the two-dimensional contour plot was oval in shape. Figure $4 \mathrm{C}, \mathrm{D}$ displays the effect of extraction temperature $\left(\mathrm{X}_{1}\right)$ and liquid-solid ratio $\left(\mathrm{X}_{2}\right)$ at an extraction time of $3 \mathrm{~h}$. The interaction effect of extraction temperature and 
liquid-solid ratio was significant on OD value ((Figure 4C, oval in shape). The interaction effect of extraction time $\left(\mathrm{X}_{3}\right)$ and liquid-solid ratio $\left(\mathrm{X}_{2}\right)$ on OD value shown in Figure 4E,F at an extraction temperature of $90^{\circ} \mathrm{C}$. It has been noted that $\mathrm{OD}$ value first enhances and then decreases at different liquid-solid ratio or extraction time. The interaction effect of extraction time and liquid-solid ratio on OD value was found non-significant (round in shape) as shown in Figure 4E. The interaction of extraction time and temperature is the most significant. While, the interaction of liquid-solid ratio and extraction temperature, and the interaction of liquid-solid ratio and extraction time is the least significant, and these results were consistent with the results of variance (Table 6.). The optimum values of extraction parameters such are extraction time, extraction temperature, and liquid-solid ratio were $2.42 \mathrm{~h}, 92.58^{\circ} \mathrm{C}$, and 25.23:1 (mL/g), respectively, and the predicted OD value calculated under the above condition was 0.741 . Noticeably, the above conditions were modified for easiness to an extraction time of $2.4 \mathrm{~h}$, extraction temperature of $93{ }^{\circ} \mathrm{C}$, and liquid-solid ratio of $25: 1(\mathrm{~mL} / \mathrm{g})$. Based on modified conditions, the concentrations of baicalin, wogonoside, and chlorogenic acid were $0.078 \mathrm{mg} / \mathrm{mL}$ (extraction yield was $15.6 \mathrm{mg} / \mathrm{g}$ ), $0.031 \mathrm{mg} / \mathrm{mL}$ (extraction yield was $6.2 \mathrm{mg} / \mathrm{g}$ ), and 0.013 $\mathrm{mg} / \mathrm{mL}$ (extraction yield was $2.6 \mathrm{mg} / \mathrm{g}$ ), respectively, and the experimental OD value was $0.759 \pm 0.09$ $(n=3)$, higher than the value obtained from non-optimum conditions. Interestingly, it was found that the model was fit and reliable to forecast the overall indexes of extraction process from L. japonicae Flos and Scutellariae Radix due to only slight deviation (2.44\%) from the true value (Table 7$)$.

Table 5. Box-Behnken design and the extraction yield.

\begin{tabular}{|c|c|c|c|c|c|c|c|}
\hline \multirow{2}{*}{ No. } & \multirow{2}{*}{$\mathrm{X}_{1}\left({ }^{\circ} \mathrm{C}\right)$} & \multirow{2}{*}{$\mathrm{X}_{2}(\mathrm{ml} / \mathrm{g})$} & \multirow{2}{*}{$X_{3}(\mathrm{~h})$} & \multicolumn{3}{|c|}{ Extraction Ratio (mg/g) } & \multirow{2}{*}{ OD } \\
\hline & & & & Baicalin & Wogonoside & Chlorogenic Acid & \\
\hline 1 & 80 & 20 & 3 & 8.46 & 4.58 & 2.54 & 0 \\
\hline 2 & 100 & 20 & 3 & 17.02 & 5.24 & 1.90 & 0.39 \\
\hline 3 & 80 & 30 & 3 & 8.46 & 7.58 & 2.52 & 0.00 \\
\hline 4 & 100 & 30 & 3 & 17.30 & 5.34 & 1.92 & 0.41 \\
\hline 5 & 80 & 25 & 2 & 8.52 & 4.00 & 2.36 & 0.06 \\
\hline 6 & 100 & 25 & 2 & 18.58 & 3.78 & 1.74 & 0 \\
\hline 7 & 80 & 25 & 4 & 8.54 & 4.10 & 2.62 & 0.09 \\
\hline 8 & 100 & 25 & 4 & 17.10 & 5.58 & 1.98 & 0.48 \\
\hline 9 & 90 & 20 & 2 & 12.88 & 4.72 & 1.88 & 0.25 \\
\hline 10 & 90 & 30 & 2 & 14.18 & 4.94 & 1.90 & 0.31 \\
\hline 11 & 90 & 20 & 4 & 13.38 & 5.62 & 2.18 & 0.48 \\
\hline 12 & 90 & 30 & 4 & 13.44 & 5.72 & 2.22 & 0.51 \\
\hline 13 & 90 & 25 & 3 & 14.64 & 6.30 & 2.38 & 0.66 \\
\hline 14 & 90 & 25 & 3 & 15.04 & 7.04 & 2.42 & 0.75 \\
\hline 15 & 90 & 25 & 3 & 14.92 & 6.38 & 2.40 & 0.69 \\
\hline 16 & 90 & 25 & 3 & 15.44 & 6.56 & 2.44 & 0.73 \\
\hline 17 & 90 & 25 & 3 & 14.38 & 6.26 & 2.40 & 0.65 \\
\hline
\end{tabular}


Table 6. Variance analysis for response surface quadratic model.

\begin{tabular}{cccccc}
\hline Source & Sum of Squares & Degree of Freedom & Mean Square & F-Value & $p$-Value \\
\hline Model & 1.160 & 9 & 0.129 & 25.800 & $0.0001^{*}$ \\
$X_{1}$ & 0.160 & 1 & 0.160 & 32.051 & $0.0008^{*}$ \\
$X_{2}$ & 0.001 & 1 & 0.001 & 0.292 & 0.6059 \\
$X_{3}$ & 0.111 & 1 & 0.111 & 22.271 & $0.0022^{*}$ \\
$X_{1} X_{2}$ & 0.000 & 1 & 0.000 & 0.0367 & 0.8535 \\
$X_{1} X_{3}$ & 0.051 & 1 & 0.051 & 10.191 & $0.0152^{*}$ \\
$X_{2} X_{3}$ & 0.000 & 1 & 0.000 & 0.034 & 0.8599 \\
$X_{1}^{2}$ & 0.563 & 1 & 0.563 & 112.67 & $<0.0001^{*}$ \\
$X_{2}^{2}$ & 0.074 & 1 & 0.074 & 14.755 & $0.0064^{*}$ \\
$X_{3}^{2}$ & 0.131 & 1 & 0.131 & 26.175 & $0.0014^{*}$ \\
Residual & 0.035 & 7 & 0.005 & & 0.0747 \\
Lack of fit & 0.028 & 3 & 0.009 & 5.104 & 0.07 \\
Pure error & 0.007 & 4 & 0.002 & & \\
Cor total & 1.196 & 16 & & & \\
\hline
\end{tabular}

Note: * Represents statistical significance at a value of $p<0.05$.
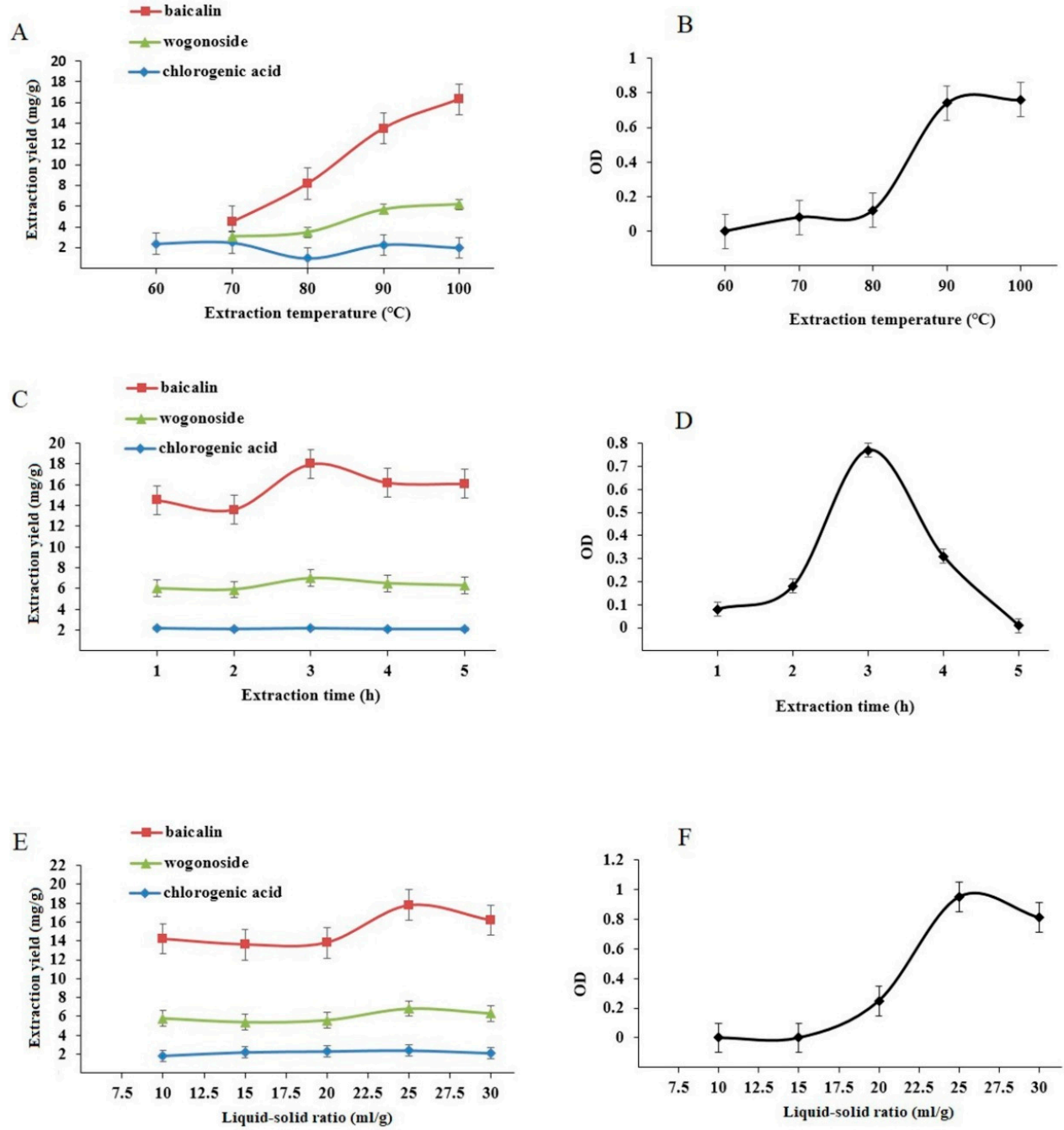

Figure 3. Effect of extraction temperature (A), extraction time (C), and liquid-solid ratio (E) on the extraction yield of baicalin, wogonoside, and chlorogenic acid and OD values (B,D,F). 

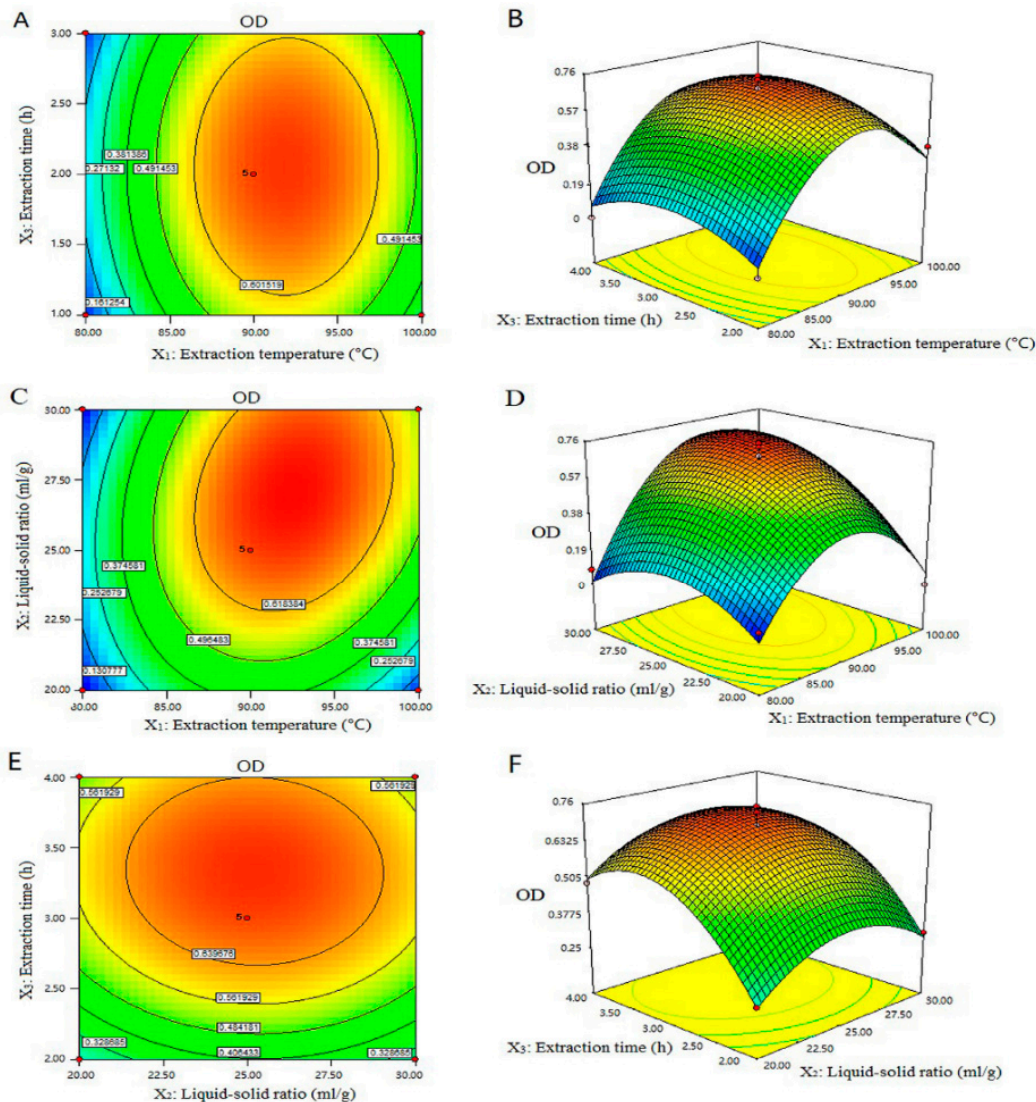

Figure 4. A-F showing response surface plots three-dimensional (3D) and contour plots two-dimensional (2D) showing the effects of different extraction parameters $\left(\mathrm{X}_{1}\right.$ : Extraction temperature, ${ }^{\circ} \mathrm{C} ; \mathrm{X}_{2}$ : Liquid-solid ratio, $\mathrm{mL} / \mathrm{g} ; \mathrm{X}_{3}$ : Extraction time, $\mathrm{h}$ ) added on the response $\mathrm{Y}$.

Table 7. Predicted and experimental values of the responses at optimum conditions.

\begin{tabular}{cccccccc}
\hline \multicolumn{2}{c}{ Optimum Condition } & \multicolumn{3}{c}{ Extraction Ratio (mg/g) } & \multicolumn{2}{c}{ OD } \\
\hline $\begin{array}{c}\text { Extraction } \\
\text { Temperature }\left({ }^{\circ} \mathrm{C}\right)\end{array}$ & $\begin{array}{c}\text { Liquid-Solid } \\
\text { Ratio }(\mathrm{mL} / \mathrm{g})\end{array}$ & $\begin{array}{c}\text { Extraction } \\
\text { Time (h) }\end{array}$ & Baicalin & Wogonoside & $\begin{array}{c}\text { Chlorogenic } \\
\text { Acid }\end{array}$ & Experimental & Predicted \\
\hline $93(92.58)$ & $25(25.23)$ & $2.4(2.42)$ & 15.6 & 6.2 & 2.6 & $0.759 \pm 0.09$ & 0.741 \\
\hline
\end{tabular}

\subsection{Effect of Extraction Cycle on Baicalin, Wogonoside, and Chlorogenic Acid Content}

Based on the optimum parameters of extraction time, extraction temperature, and liquid-solid ratio, the extraction cycle was analyzed. It has been noted that the extraction cycle from one to two significantly $(p<0.05)$ increased the yield of the three compounds (Figure 5). However, the extraction yield is slowly paced down after two. Therefore, extraction cycle two was chosen as the suitable extraction cycle to save time and reduce energy cost. The data showed that the extraction yield of chlorogenic acid was less than the other two compounds and therefore showed deviation from the other two compounds. 

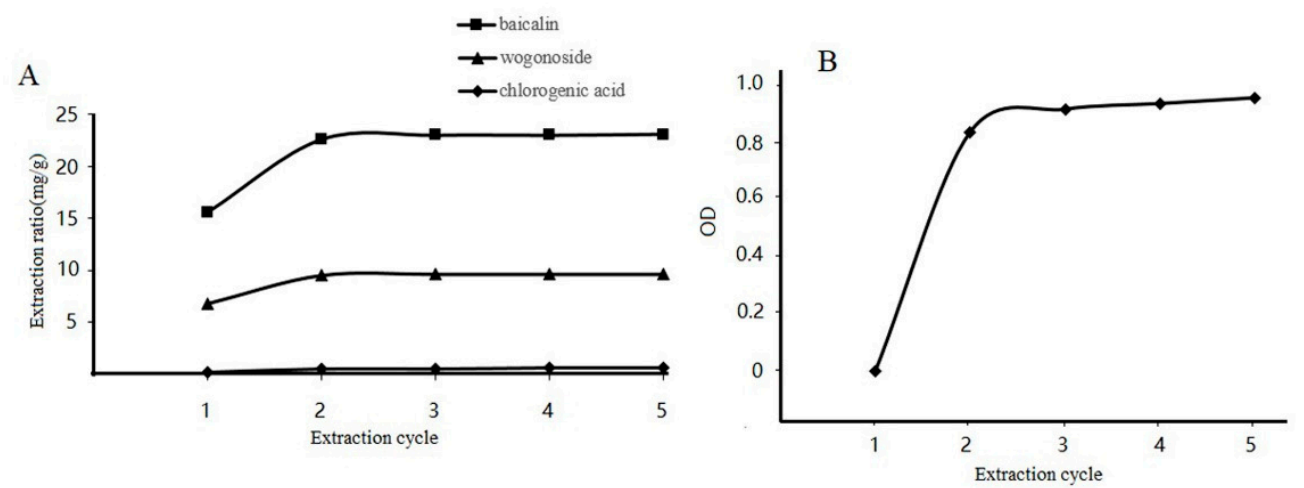

Figure 5. Effect of extraction number on the yield of baicalin, wogonoside, and chlorogenic acid. (A) shows the effect of extraction cycle on extraction yield $(\mathrm{mg} / \mathrm{g})$ of the three compounds, whereas (B) shows the obtained OD values at different extraction cycles.

\section{Discussion}

Previous studies reported that highly accurate extraction methods are very important for the development of a sensitive and reliable inexpensive extraction methods [15]. In this study, we optimized a water extraction process for baicalin, wogonoside, and chlorogenic acid. Three factors (extraction time, extraction temperature, and liquid-solid ratio) were chosen in single factor experiments which could affect the extraction process significantly. Depicting of a 3D-response surface and quadratic polynomial fitting analysis were performed to choose the final extraction conditions for the three tested parameters. In general, lack of fit test was employed to analyze the variation in the data around the fitted model [16]. Previous report demonstrated that proper preparation of samples gives good recovery results [17]. Ekberg et al. (2008) demonstrated that choosing of an extraction solvent which is easily available and cheap plays a crucial role in optimization of an extraction process [18]. In another study, Sheng et al. (2018) used ethanol as an extraction solvent and examined the effect of different concentration of ethanol $(0 \%, 20 \%, 40 \%, 60 \%, 80 \%$, and $100 \%)$ on total phenolic content from Terminalia chebula Retz. fruits using response surface methodology [19]. They noted that $60 \%$ ethanol gives higher extraction yield than other tested concentrations of ethanol. While, in the present study, water was used as an extraction solvent due to easy availability and cheap price relative to other solvents. It can be seen from the methodological investigation that the RSD\% value of each test result is less than 3\%, indicating that the method has high precision value, good repeatability, recovery, and stability. In addition, the data showed that the optimized method is reproducible and precise in terms of linearity, precision, and stability experiments. Recently, more scientists have studied the methods of simultaneous quantification of various components in traditional Chinese medicine [20]. Therefore, in this study, we also established and optimized a simultaneous extraction method for wogonoside, chlorogenic acid, and baicalin. It is often necessary to examine multiple levels of multiple factors for optimization of an extraction process. In order to reduce the number of trials and to investigate the interaction between factors, uniform design and orthogonal design methods are often used in China to design experiments [21]. It is worthy to mention that linear model is mostly used in the extraction process. The larger the linear correlation coefficient, the farther the factor level is from the optimal value [22]. In the present experiments, Box-Behnken design was employed to optimize the process due to the advantages of reproducibility test at the center point which can improve the accuracy of mathematical model prediction. The amount of information obtained by the test is larger, more intuitive, and more suitable for exploratory experimental research and there is no trial frequency and cost [23-25]. It has been reported previously that medicinal plants have been used against various illnesses, and flavonoids have efficient antioxidant and anti-inflammatory activities, etc. [26]. Based on the pharmacological uses of baicalin and wogonoside (flavonoids) and chlorogenic acid (polyphenol), we optimized an efficient and simultaneous water extraction process. Nevertheless, further research is 
needed to optimize the process for other flavonoids. In summary, the results of the study indicated that the method is fully validated and optimized by Box-Behnken design approach. The optimized method will be an efficient tool for the extraction of traditional Chinese medicine such as wogonoside, chlorogenic acid, and baicalin at the same time. The data showed that the optimized conditions are: Liquid to solid ratio $25: 1(\mathrm{~mL} / \mathrm{g})$, extraction temperature $93^{\circ} \mathrm{C}$, extraction time $2.4 \mathrm{~h}$, and the extraction cycle two. Under these conditions, the extraction yield of baicalin, wogonoside, and chlorogenic acid are an efficient alternative to expensive solvent extraction methods. Moreover, the optimized method was inexpensive, feasible, stable, and reliable.

Author Contributions: Conceptualization, J.L.; Methodology, R.W.; Software, Z.S.; Validation, Z.W.; Formal Analysis, Z.Y.; Investigation, R.W.; Resources, J.L. and Z.S.; Data Curation, Z.S.; Writing-Original Draft Preparation, M.I.; Writing-Review \& Editing, M.I. and C.C.; Supervision, J.L.; Funding Acquisition, J.L. and C.C.

Funding: This study was supported by the National Natural Science Foundation of China (31772801 and 31973005), project of Youth Innovative Talent Training Program in Heilongjiang Province (UNPYSCT-2018146), and Academic Backbone Project of Northeast Agricultural University (18XG23).

Conflicts of Interest: All authors declared no conflicts of interest.

\section{References}

1. Shang, X.; He, X.; He, X.; Li, M.; Zhang, R.; Fan, P.; Zhang, Q.; Jia, Z. The genus Scutellaria an ethnopharmacological and phytochemical review. J. Ethnopharmacol. 2010, 128, 279-313. [CrossRef] [PubMed]

2. Li-Weber, M. New therapeutic aspects of flavones: The anticancer properties of Scutellaria and its main active constituents Wogonin, Baicalein and Baicalin. Cancer Treat. Rev. 2009, 35, 57-68. [CrossRef] [PubMed]

3. Zhao, Q.; Chen, X.Y.; Martin, C. Scutellaria baicalensis, the golden herb from the garden of Chinese medicinal plants. Sci. Bull. 2016, 61, 1391-1398. [CrossRef] [PubMed]

4. Xue, T.H.; Roy, R. Studying traditional Chinese medicine. Science 2003, 300, 740-741. [CrossRef]

5. Zhang, L.; Ren, Y.; Yang, C.; Guo, Y.; Zhang, X.; Hou, G.; Guo, X.; Sun, N.; Liu, Y. Wogonoside ameliorates lipopolysaccharide-induced acute lung injury in mice. Inflammation 2014, 37, 2006-2012. [CrossRef]

6. Kovacs, G.; Kuzokina, I.N.; Szoke, E.; Kursinszki, L. HPLC determination of flavonoids in hairy-root cultures of Scutellaria baicalensis Georgi. Chromatographia 2004, 60, S81-S85. [CrossRef]

7. Zhang, T.; Mi, M.T.; Tang, Y.; Zhao, J. The extraction of polyphenol contents of Chaenomeles sinensis and its effect on scavenging DPPH radical. Acta Nutr. Sin. 2007, 29, 485-489.

8. Cai, H.; Cao, G.; Li, L.; Liu, X.; Ma, X.-Q.; Tu, S.-C.; Lou, Y.-J.; Qin, K.-M.; Li, S.-L.; Cai, B.-C. Profiling and characterization of volatile components from non-fumigated and sulfur-fumigated flos Lonicerae japonicae using comprehensive two-dimensional gas Chromatography time-of-flight mass spectrometry coupled with chemical group separation. Molecules 2013, 18, 1368-1382. [CrossRef]

9. Wu, M.Z.; Xu, Y.L.; Li, F.; Wei, P.; Wang, Z.; Luo, Z.P.; Wang, R.; Zhang, J.F.; Lin, F.C.; Yang, J. Cloning and expression analysis of chlorogenic acid biosynthetic gene NtHQT1 from Nicotiana tabacum. Tob. Sci. Technol. 2015, 48, 1-6. [CrossRef]

10. Nascu-Briciu, R.D.; Cobzac, S.C.; Baciu, S. Optimum ultrasound assisted extraction conditions of some flavonoids from green tea leaves. Control quality of green tea product by TLC fingerprinting. Anal. Lett. 2011, 44, 2865-2875. [CrossRef]

11. Repolles, C.; Herrero-Martinez, J.M.; Rafols, C. Analysis of prominent flavonoid aglycones by high-performance liquid chromatography using a monolithic type column. J. Chromatogr. A. 2006, 1131, 51-57. [CrossRef] [PubMed]

12. Al-Temimi, A.; Choudahry, R. Determination of antioxidant activity in different kinds of plants in vivo and in vitro by using diverse technical methods. J. Nutr. Food Sci. 2013, 3, 1-6. [CrossRef]

13. Herrera, M.C.; de Castro, M.D.L. Ultrasound-assisted extraction of phenolic compounds from strawberries prior to liquid chromatographic separation and photodiode array ultraviolet detection. J. Chromatogr. A. 2005, 1100, 1-7. [CrossRef] [PubMed]

14. Bajer, T.; Adam, M.; Galla, L.; Ventura, K. Comparison of various extraction techniques for isolation and determination of isoflavonoids in plants. J. Sep. Sci. 2007, 30, 122-127. [CrossRef] [PubMed] 
15. Elerath, J.; Pecht, M. A highly accurate method for assessing reliability of redundant arrays of inexpensive disks (RAID). IEEE Trans. Comput. 2009, 58, 289-299. [CrossRef]

16. Trinh, T.K.; Kang, L.S. Application of response surface method as an experimental design to optimize coagulation test. Environ. Eng. Res. 2010, 15, 63-70. [CrossRef]

17. Cui, X.; Muhammad, I.; Li, R.; Jin, H.; Guo, Z.; Yang, Y.; Hamid, S.; Li, J.; Cheng, P.; Zhang, X. Development of a UPLC-FLD Method for Detection of Aflatoxin B1 and M1 in Animal Tissue to Study the Effect of Curcumin on Mycotoxin Clearance Rates. Front. Pharmacol. 2017, 8, 650. [CrossRef]

18. Ekberg, C.; Fermvik, A.; Retegan, T.; Skarnemark, G.; Foreman, M.R.S.; Hudson, M.J.; Englund, S.; Nilsson, M. An overview and historical look back at the solvent extraction using nitrogen donor ligands to extract and separate An (III) from Ln(III). Radiochim. Acta 2008, 96, 225-233. [CrossRef]

19. Sheng, Z.; Zhao, J.; Muhammad, I.; Zhang, Y. Optimization of total phenolic content from Terminalia chebula Retz. fruits using response surface methodology and evaluation of their antioxidant activities. PLoS ONE 2018, 13, e0202368. [CrossRef]

20. Feng, J.; Xu, W.; Tao, X.; Wei, H.; Cai, F.; Jiang, B.; Chen, W. Simultaneous determination of baicalin, baicalein, wogonin, berberine, palmatine and jatrorrhizine in rat plasma by liquid chromatography-tandem mass spectrometry and application in pharmacokinetic studies after oral administration of traditional Chinese medicinal preparations containing scutellaria-coptis herb couple. J. Pharm. Biomed. Anal. 2010, 53, 591-598.

21. Ni, H.; Wu, Z.; Muhammad, I.; Lu, Z.; Li, J. Optimization of baicalin water extraction process from Scutellaria baicalensis (a traditional Chinese medicine) by using orthogonal test and HPLC. Rev. Bras. Farmacogn. 2017. [CrossRef]

22. Sun, Y.; Li, T.; Yan, J.; Liu, J. Technology optimization for polysaccharides (POP) extraction from the fruiting bodies of Pleurotus ostreatus by Box-Behnken statistical design. Carbohydr. Polym. 2010, 80, 242-247. [CrossRef]

23. Zhang, T.F.; Yang, J.F.; Lin, D.K.J. Small Box-Behnken design. Stat. Probab. Lett. 2011, 81, 1027-1033. [CrossRef]

24. Bezerra, M.A.; Santelli, R.E.; Oliveira, E.P.; Villar, L.S.; Escaleira, L.A. Response Surface Methodology (RSM) as a Tool for Optimization in Analytical Chemistry. Talanta 2008, 76, 965-977. [CrossRef]

25. Pinho, C.; Melo, A.; Mansilha, C.; Ferreira, I.M.P.L.V.O. Optimization of Conditions for Anthocyanin Hydrolysis from Red Wine Using Response Surface Methodology (RSM). J. Agric. Food Chem. 2011, 59, 50-55. [CrossRef]

26. Altemimi, A.; Watson, D.G.; Kinsel, M.; Lightfoot, D.A. Simultaneous extraction, optimization, and analysis of flavonoids and polyphenols from peach and pumpkin extracts using a TLC-densitometric method. Chem. Central J. 2015, 9, 39. [CrossRef]

(C) 2019 by the authors. Licensee MDPI, Basel, Switzerland. This article is an open access article distributed under the terms and conditions of the Creative Commons Attribution (CC BY) license (http://creativecommons.org/licenses/by/4.0/). 